\title{
Claude Syndrome 'Plus' in an Adolescent
}

\author{
Michael S. Salman, Nicole Froese, Paul Shuckett, Jens Wrogemann, David D. Eisenstat
}

Can. J. Neurol. Sci. 2006; 33: 430-432

A 13-year-old boy presented to the pediatric emergency department with a five-day history of headache, vomiting and double vision. He had previously been well. He was not on any medications. His birth, developmental and family history was unremarkable. He resided with his family in rural Manitoba, Canada.

On examination he was fully oriented. His GCS was 15 . His general examination was unremarkable. His blood pressure was $131 / 77$ and heart rate 67 beats per minute. On inspection, he had a left head tilt and left-sided ptosis. His palpebral fissure width, with the eyes in primary position, measured $8 \mathrm{~mm}$ on the right and $5 \mathrm{~mm}$ on the left. His best-corrected monocular near visual acuity was $20 / 20$ on the right and $20 / 30^{-2}$ on the left, using a pinhole. With his glasses, his near visual acuity was 20/30 on the right and 20/200 on the left. His visual fields were full on confrontation. His right pupil was $3 \mathrm{~mm}$ and left pupil was $4 \mathrm{~mm}$ in diameter in the light. The left pupil was minimally reactive to direct and indirect light, while the right pupil reacted sluggishly and partially to direct and indirect light (i.e., the left pupil was less responsive to stimulation with light than the right pupil). Extraocular movements of the left eye were restricted to $20 \%$ of normal on upgaze, $30 \%$ on downgaze, $80 \%$ on adduction, but
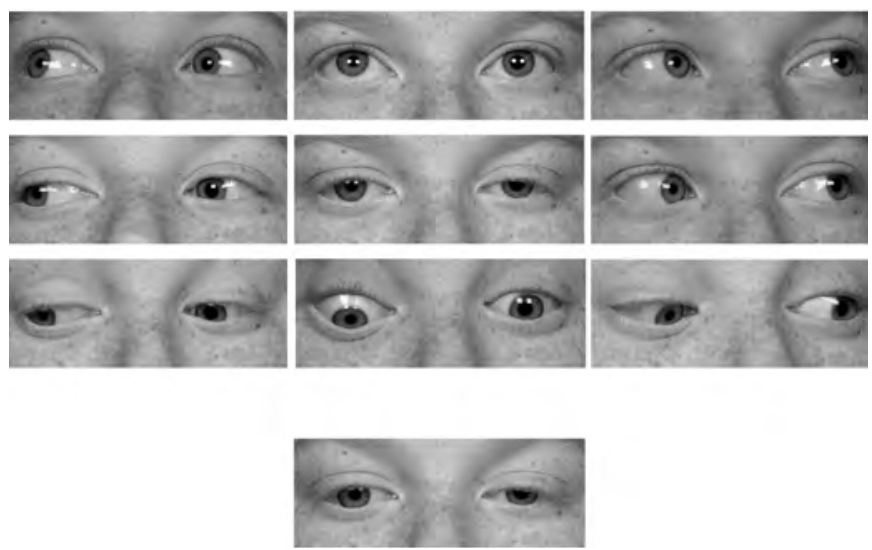

Figure 1. The upper photograph shows the patient's eyes in nine directions of gaze. In neutral gaze, ptosis of the left eye and a larger papillary size in comparison with the right pupil can be seen. There is bilateral restriction of eye movements on upgaze affecting the left more than the right eye. In addition, there is partial restriction of the left eye movement on downgaze and on upgaze following adduction. Adduction of the left eye is restricted to a lesser extent. The lower photograph was taken while the patient was asked to look at a near object $25 \mathrm{~cm}$ away. There is a lack of convergence and pupillary constriction. moved fully on abduction. Extraocular movements of the right eye were restricted to $40 \%$ of normal on upgaze, with the rest being full (Figure 1). The limitations of eye movements were not overcome by the oculo-cephalic maneuver. Bell's phenomenon was absent. These findings indicated that his eye movements' restrictions were caused by a lower motor neuron lesion. He could not converge, even though he was able to adduct his left eye $80 \%$ of normal on rightward gaze, and his pupils did not constrict when asked to converge his eyes. Within the restricted range of eye movements, his smooth pursuit (slow eye movements) and saccades (fast eye movements) were normal. He had binocular oblique diplopia maximal on left downward gaze. In that position, his left hypertropia was maximal and measured 18 prism diopters. Fundoscopy demonstrated blurred optic discs' margins. Other cranial nerves were normal. Upper and lower limbs tone, power, coordination, deep tendon reflexes and sensation were normal. He had a slightly ataxic gait.

Magnetic resonance imaging (MRI) of the brain (Figure 2) showed a hematoma in the midbrain, likely a cavernoma (cavernous hemangioma) and obstructive hydrocephalus caused by compression of the aqueduct of Sylvius. Blood work and coagulation factors were normal.

The patient underwent an endoscopic third ventriculostomy. After surgery, the patient's headaches and vomiting resolved. His cavernoma was managed conservatively and eye patching was recommended for his diplopia. He was discharged home four days later. Six weeks later, his optic discs were normal but the rest of the eye exam findings persisted. In addition, he developed dysarthria and right upper limb rebound, intention tremor, dysdiadochokinesis and dysmetria. He had right heel-shin incoordination and an ataxic gait. A repeat MRI showed further blood leakage into the midbrain. The cerebellar signs resolved five months later.

The neuro-ophthalmologic findings suggested an absent near response (i.e., lack of convergence, miosis, and accommodation). His near visual acuity was degraded because he could not accommodate fully. His pupils did not constrict and he could not converge his eyes when asked to look at a near object. The midbrain is important for processing the near

From the Sections of Pediatric Neurology (MSS), Ophthalmology (PS), Pediatric Radiology (JW), Hematology/Oncology (DDE), Children's Hospital; Departments of Pediatrics \& Child Health (MSS, DDE), Human Anatomy \& Cell Science (DDE), Radiology (JW) and Ophthalmology (PS, DDE), Faculty of Medicine (NF), University of Manitoba, Winnipeg, MB, Canada.

ReCEIVED MAY 1, 2006. ACCEPTED IN FinAl FORM SEPTEMBER 2, 2006.

Reprint requests to: Michael S. Salman, Section of Paediatric Neurology, AE 108, Harry Medovy House, Children's Hospital, 820 Sherbrook St., Winnipeg, Manitoba, R3A 1R9, Canada. 


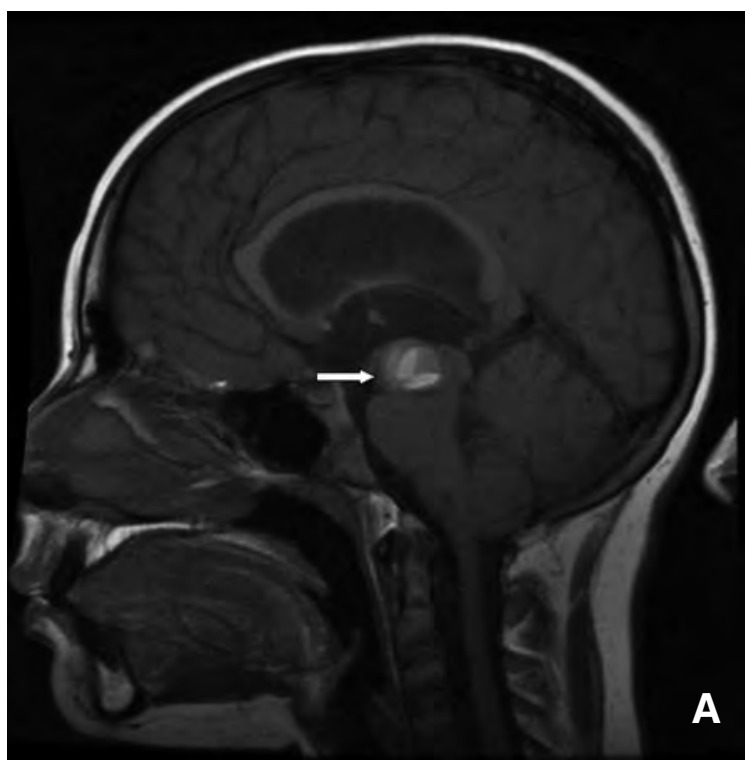

Figure 2.

(a) Sagittal T1 weighted MRI image (TR 367, TE 14) showing obstructive hydrocephalus caused by a lesion with mixed signal intensity hematoma in the midbrain (arrow). These findings are consistent with a cavernoma with hemosiderin.

(b) Axial T2 weighted MRI image (TR 2350, TE 111) showing mixed signal intensity hematoma in the midbrain with hemosiderin/ blood level (arrow), dilated third ventricle (*) and temporal horns of the lateral ventricles.

(c) Axial T2 weighted MRI image (TR2350, TE 111) showing a hypointense hemosiderin ring surrounding the cavernoma (upper arrow), five months after presentation. The lower arrow shows the aqueduct of Sylvius. There is a low signal flow void in $3 r d$ ventricle from the patent 3rd ventriculostomy (*). The lateral ventricles are reduced in size.
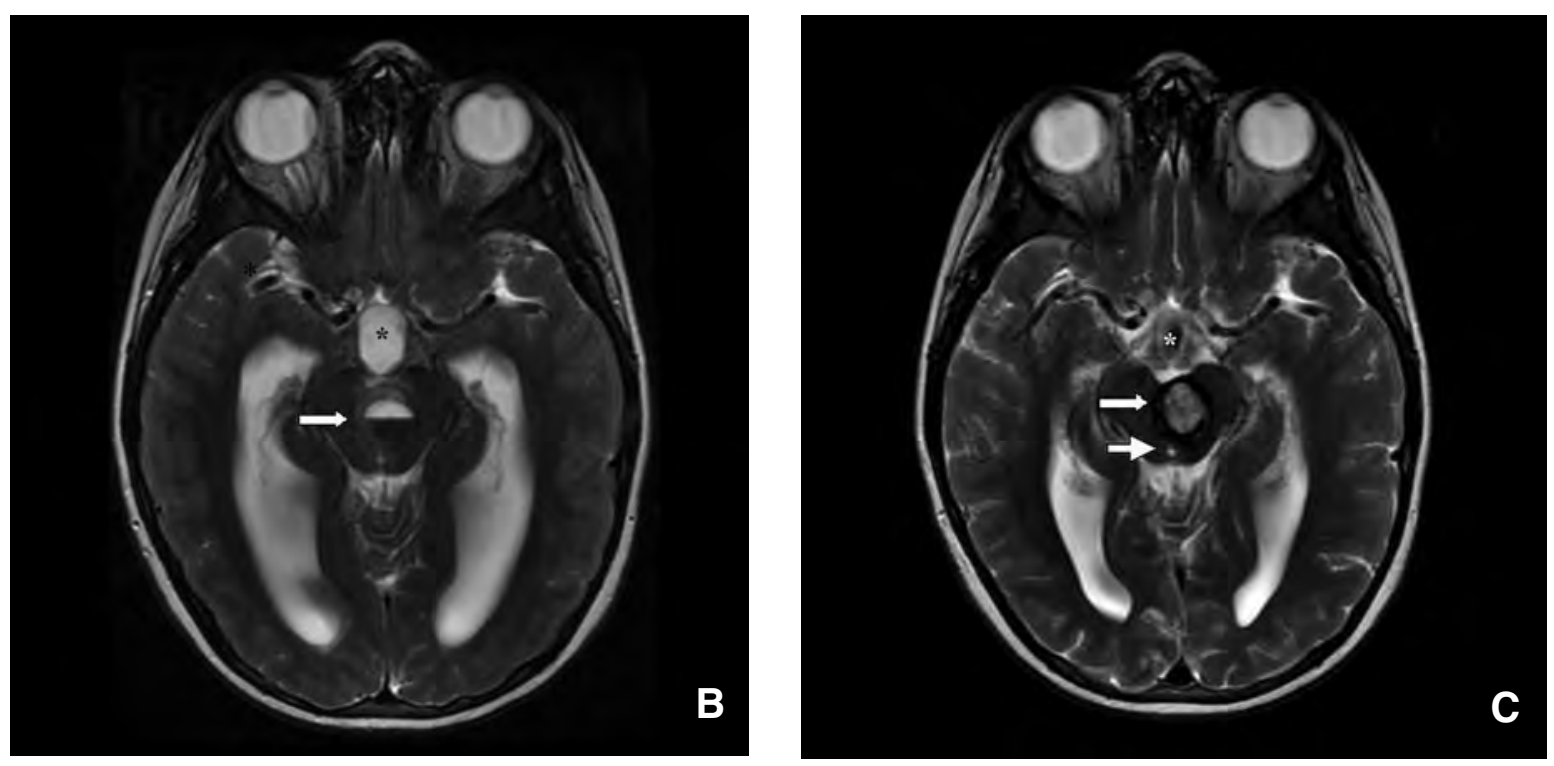

response. His bilateral abnormal pupillary response to light suggested bilateral (left more than right) involvement of any or a combination of the following: the pretectal nuclei, the EdingerWestphal nuclei or the tracts connecting them, because the direct and consensual light responses were affected bilaterally. The pretectal nuclei, which are innervated by the optic tract, lie in the midbrain behind the aqueduct and each nucleus projects bilaterally to the Edinger-Westphal nuclei. The latter lie on the superior aspect of the III (oculomotor) nuclear complex, ${ }^{1}$ and supply the pupils with parasympathetic nerve fibers.

The patient also had a left partial nuclear III nerve paresis. Nuclear III nerve palsies are rare in the general population and unreported in pediatrics. The III nerve nucleus is located in the upper midbrain, anterior to the Sylvian aqueduct. It innervates four extraocular muscles and the levator palpebral superioris

muscle as well as supplying presynaptic parasympathetic fibers for pupillary constriction and lens accommodation. The III nerve nuclear complex is divided into subnuclei. The medial rectus, inferior rectus and inferior oblique subnuclei send efferent nerve fibers ipsilaterally to the extraocular muscles, whereas the superior rectus subnuclei send efferent nerve fibers across the midline and into the contralateral III nerve nucleus, which in turn send projections to the superior rectus muscle. ${ }^{2}$ Therefore, each superior rectus muscle is innervated by the contralateral III nerve nucleus. The levator palpebrae superioris muscles are innervated by a single region, the central caudal nucleus, which lies in the posterior superior aspect of the III nerve nucleus. The nerve supply of each muscle contains both crossed and uncrossed nerve fibers. Thus a unilateral lesion of the third nerve nucleus causes bilateral limitation of upward gaze and asymmetric ptosis, 
which is worse ipsilaterally. The inferior and superior recti, and inferior oblique muscles will also be weak with a dilated pupil ipsilateral to the lesion. The patient did not have an obvious right-sided ptosis suggesting that it may have been either subtle or minimally affected by the lesion. His initial slightly ataxic gait was likely related to hydrocephalus.

Compression and hemorrhage into the surrounding parenchyma from the midbrain cavernoma caused an unusual combination of neuro-ophthalmological signs. The left III nerve nuclear complex, midbrain neurons that encode the near triad, and the pretectal/ Edinger-Westphal nuclei or their projections were all involved. With the subsequent bleed and the development of right-sided cerebellar signs on follow-up, the diagnosis of Claude syndrome 'plus' was made. The main features of Claude syndrome consists of a combination of ipsilateral III nerve palsy and contralateral ataxia, due to a lesion involving the crossing of the superior cerebellar peduncle tract in the midbrain at the level of the adjacent III nerve fascicles., Claude described this syndrome in 1912 in a patient with midbrain infarction that involved the decussation of the superior cerebellar peduncle, the medial part of the red nucleus and III nerve fascicles. ${ }^{3-5}$ The midbrain lesion in our case is large and extends more dorsally than the midbrain infarct described in Claude's case because the III nerve nucleus and pretectal/ Edinger-Westphal nuclei and their connections were also involved in our case; hence, the designation of Claude syndrome 'plus'.

Nuclear III nerve palsy is rare. Common causes of fascicular III nerve palsy in adults are vascular/ diabetic, aneurysm, trauma, and neoplasm. If the extraocular movements or pupillary responses are impaired but not absent, the palsy is considered partial. In children, the three major causes are trauma $(40.0 \%)$, neoplasm $(17.1 \%)$, and undetermined $(14.3 \%)$, followed by postoperative $(8.6 \%)$, ophthalmoplegic migraines $(8.6 \%)$, and meningitis $(2.4 \%){ }^{6}$ Another study found that after congenital nerve palsies, trauma was the leading cause followed by neoplasm then vascular causes. ${ }^{7}$

Cavernous malformations (CM), also called cavernous angiomas, cavernomas or cavernous hemangiomas, are vascular malformations found in the central nervous system and made up of a compact mass of vessels. They are described as 'mulberry' in appearance due to their color and lobulation. Microscopically, $\mathrm{CM}$ are made of thin, dilated capillaries, comprised of an endothelial lining and a fibrous adventitia, but no elastic fibers. ${ }^{8,9}$ In the general population, the incidence of cavernomas is approximately $0.4 \%$ and most commonly occur somewhere between the second and fifth decade. However, up to $25 \%$ of those presenting with CM may be younger than 18 years old. ${ }^{8,10}$ There is an inherited and sporadic type of CM. Those with the sporadic form are more likely to have a single lesion. ${ }^{11}$

Cavernous malformations can present with different manifestations, often according to the location of the lesion. Supratentorial lesions most commonly present with seizures whereas brainstem lesions are more likely to have a sudden onset of focal neurological deficits. ${ }^{11}$ Cavernous malformations can present with hemorrhage at any site, resulting in symptoms ranging from mild to death. ${ }^{8}$ Patients who present with deficits due to hemorrhage often have poor resolution of symptoms and are at risk of repeat hemorrhages causing further neurological deficits. ${ }^{1}$ Most CM have a potential for growth, which separates them from other vascular malformations. ${ }^{12}$ The mechanism of this growth is the result of repeated hemorrhages, formation of a clot and growth of new vasculature. In children, there is a significant risk of rapid lesion growth and developing new lesions; therefore children need to receive detailed and regularly scheduled follow-up. ${ }^{10}$

Diagnosis of CM is most effective with MRI, although CT can also be used. Cavernous malformations can often be incidental findings without associated symptoms; these can be treated conservatively and often need no therapy at all. Surgical risks may not be justified as data suggests that the risk of symptomatic hemorrhage is $<1 \%$ per year. ${ }^{11}$ Treatment is more often reserved for those with multiple episodes of hemorrhage or intractable seizures. There has been evidence that stereotactic radiosurgery is effective in decreasing the frequency of seizures and risk of hemorrhage; however, the long-term effects of radiosurgery are not yet fully defined, especially in children. ${ }^{9}$

In summary, sudden onset of headache and diplopia warrant a prompt neurological assessment. Cerebral vascular anomalies, although rare, should be included in the differential diagnosis of any child presenting with these symptoms and signs. This case is instructive and reveals the importance of a careful neuroophthalmological examination in helping to localize brain lesions and also provides a way for monitoring the patient on follow-up.

\section{ACKNOWLEDGEMENT}

The authors thank Dr. Patrick MacDonald for his excellent neurosurgical care of this patient. We also thank the patient and his family for their consent to publish the case report.

\section{REFERENCES}

1. Carlow T. Oculomotor nerve. In: Aminoff MJ, Daroff RB, editors. Encyclopedia of the neurological sciences. San Diego: Elsevier; 2003: 3, p. 660-2.

2. Bienfang DC. Crossing axons in the third nerve nucleus. Invest Ophthalmol. 1975;14(12):927-31

3. Liu GT, Crenner CW, Logigian EL, Charness ME, Samuels MA. Midbrain syndromes of Benedikt, Claude, and Nothnagel: setting the record straight. Neurology. 1992;42(9):1820-2.

4. Seo SW, Heo JH, Lee KY, Shin WC, Chang DI, Kim SM, et al. Localization of Claude's syndrome. Neurology. 2001; 57(12):2304-7.

5. Claude H, Loyez M. Ramollissement du noyau rouge. Rev Neurol. (Paris) 1912;24:49-51.

6. Kodsi SR, Younge BR. Acquired oculomotor, troclear and abducens cranial nerve palsies in pediatric patients. Am J Ophthalmol. 1992;114(5):568-74.

7. Schumacher-Feero LA, Yoo KW, Solari FM, Biglan AW. Third cranial nerve palsy in children. Am J Ophthalmol. 1999;128(2):216-21.

8. Brown RD, Flemming KD, Meyer FB, Cloft HJ, Pollock BE, Link ML. Natural history, evaluation and management of intracranial vascular malformations. Mayo Clinic Proc. 2005;80(2):269-81.

9. Smith ER, Butler WE, Ogilvy CS. Surgical approaches to vascular anomalies of the child's brain. Neurology. 2002;15:165-71.

10. Frim DM, Scott RM. Management of cavernous malformations in the pediatric population. Neurosurg Clin N Am. 1999;10(3):5138.

11. Spetzler R, Zabramski J. Cavernous malformations. In: Aminoff MJ, Daroff RB, editors. Encyclopedia of the neurological sciences. San Diego: Elsevier; 2003, 1, p. 532-8.

12. Giulioni M, Acciarri N, Padovani R, Frank F, Galassi E, Gaist G. Surgical management of cavernous angiomas in children. Surg Neurol. 1994;42(3):194-9. 